Sādhanā Vol. 38, Part 1, February 2013, pp. 109-121. (C) Indian Academy of Sciences

\title{
Modelling spatial density using continuous wavelet transforms
}

\author{
D SUDHEER REDDY ${ }^{1, *}, \mathrm{~N}_{\text {GOPAL REDDY }}^{2}$ and \\ A K ANILKUMAR ${ }^{3}$
}

\author{
${ }^{1}$ Digital Mapping and Modelling Division, Advanced Data Processing Research \\ Institute, Secunderabad 500 009, India \\ ${ }^{2}$ Department of Mathematics, Osmania University, Hyderabad 500 007, India \\ ${ }^{3}$ Applied Mathematics Division, Vikram Sarabai Space Centre, \\ Trivandrum 695 022, India \\ e-mail: sudheer@adrin.res.in,donsudhir@gmail.com
}

MS received 26 April 2011; revised 25 June 2012; accepted 12 October 2012

\begin{abstract}
Due to increase in the satelite launch activities from many countries around the world the orbital debris issue has become a major concern for the space agencies to plan a collision-free orbit design. The risk of collisions is calculated using the in situ measurements and available models. Spatial density models are useful in understanding the long-term likelihood of a collision in a particular region of space and also helpful in pre-launch orbit planning. In this paper, we present a method of estimating model parameters such as number of peaks and peak locations of spatial density model using continuous wavelets. The proposed methodology was experimented with two line element data and the results are presented.
\end{abstract}

Keywords. Space debris; wavelets; Mexican hat; Laplace distribution; random search; parameter estimation.

\section{Introduction}

The utilization of space around earth has incited many countries to participate and compete with each other to make the best use of this natural asset. In the process a large number of man made objects in the form of artificial satellites are added to the space environment which already houses natural meteroids and other interplanetary dust. The orbiting objects which no longer serve any purpose are termed orbital debris. In-orbit collisions deliberate or unintentional could become the primary source of new space debris. As the demand for space-based systems for remote sensing, communications and navigation are ever increasing, it is important to understand the nature of the threat that orbital debris pose to operational satellites and take appropriate steps to ensure the sustainable development of near-Earth space.

*For correspondence 
Figure 1 shows a snap shot of Earth's space debris environment taken by European Space Agency in 1997. Figure 2 shows the evolution of space debris since 1957-2011. (Source ODQN, NASA).

The orbits that are at a distance of $200 \mathrm{~km}-2000 \mathrm{~km}$ are referred to as low Earth orbits (LEO), and $24,000 \mathrm{~km}$ to $36,000 \mathrm{~km}$ are called geostationary Earth orbits (GEO). Orbital debris can be detected by ground-based radars, but their visibility is restricted by its power, size of the object and its distance from the Earth. Objects of size $10 \mathrm{~cm}$ in LEO and $1 \mathrm{~m}$ in GEO are detectable along with its trajectory. Optical telescopes also provide measurement of debris objects to a size of less than $5 \mathrm{~cm}$ in LEO and $60 \mathrm{~cm}$ in GEO. The various parameters describing the position of the scanned object at the time of scan (epoch), security classification, nature of debri (e.g., intact or debris object, spacecraft- or rocket-related), effect of perturbations on object's motion are provided in the form of two line elements shortly termed as TLE. A detailed description of TLEs and their derivatives are presented in Vallado (2001).

The complete picture of the orbital debris environment is not available with any kind of in situ and remote measurements. Modelling space debris environment provides a mathematical description of the spatio-temporal distribution and physical characteristics of man-made objects in space. These models may be deterministic in nature governed by physical laws of gravitation or statistical in nature governed by sample observation or a combination of both.

These models are used to simulate the current and future space debris environment. The serious efforts put forward by space agencies such as NASA, ESA, led to the development of ORDEM (Orbital Debris Engineering Model) and MASTER (Meteoroid and Space Debris Terrestrial Environment Reference model). These models have evolved over two decades by capitalizing proven characterizations between various parameters describing the debris environment such as number of objects vs inclination, spatial density vs altitude, size of objects vs velocity, flux vs velocity, etc. (Klinkrad 2006).

In the present work, we focus on spatial density models. Spatial density refers to the number of debri objects per cubic kilometer in space around Earth. Spatial density models are useful in understanding the long-term likelihood of a collision in a particular region of space and also

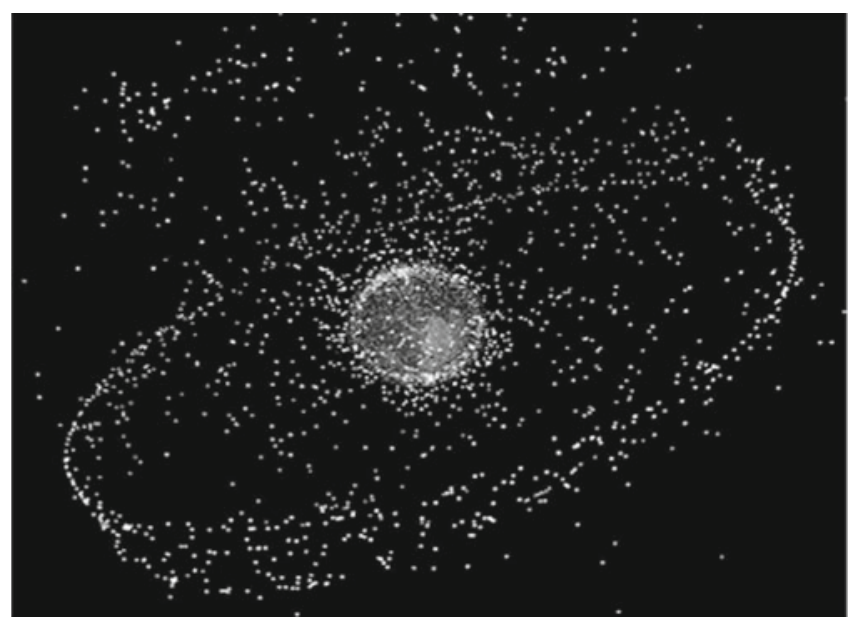

Figure 1. Global snapshot of the catalogue population in the year 1997 (source: European Space Agency (ESA)). 


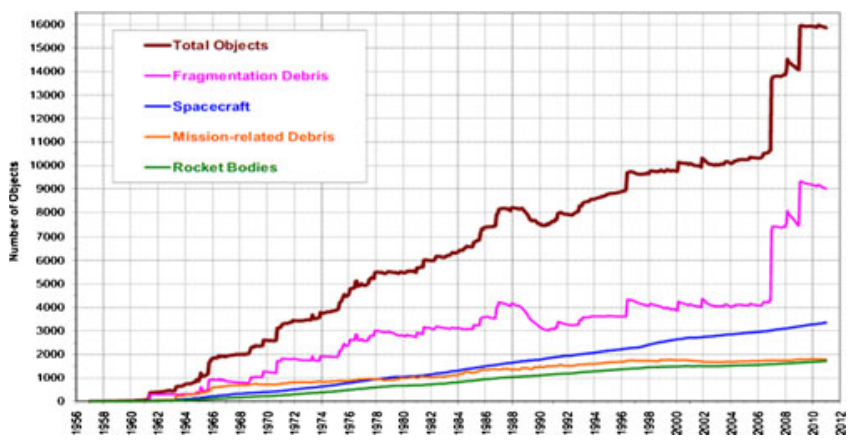

Figure 2. Evolution of space debris environment in number of objects per year (source: ODQN, NASA).

helpful in pre-launch orbit planning. Ananthasayanam et al (2006), have shown that the number density ' $n$ ' in LEO can be represented by a mixture of Laplace distributions. Anilkumar $\&$ Sudheer Reddy (2009) used these models for statistical conjunction analysis by classifying the debris population in low Earth orbits into three inclination regions viz., $0^{\circ}-180^{\circ}, 95^{\circ}-105^{\circ}$, $98^{\circ}-99^{\circ}$. Since most of the earth observing satellites are in near polar orbits, conjunctions in the regions $95^{\circ}-105^{\circ}$ and $98^{\circ}-99^{\circ}$ are critically analysed. Conjunction analysis considering Earth's oblateness and atmospheric drag was reported by Sudheer Reddy \& Anilkumar (2008). When the probability of conjunction is very high and the miss distance between active satellite and debri object is less then a threshold, a collision avoidance maneuver is employed. Some maneuvers adopted during 2010 are reported in Liou (2010).

The presence of abrupt variations/peaks in any data carries important information, it gives an indication of some transient behaviour of the underlying physical phenomenon. A local window based search for peaks is sensitive to noise and do not differentiate strong and weak peaks. Methods based on using Fourier transforms employ matching approaches to compare the frequency values within a fixed window with a reference peak and a similarity measure. Both these methods are dependent on size of the window and/or prior knowledge about the nature of the peak. Classification, detection and measurement of such peaks/singularities using wavelets was given by Mallat \& Hwang (1992). Continuous Wavelet Transforms (CWT) provides a redundant and finely detailed description of a data/signal in terms of both time and frequency. CWTs are particularly helpful in tackling problems involving signal identification and detection of hidden transients (hard to detect, short-lived elements of a signal).

Parameter estimation becomes important in many stochastic models for calculating parameters of the proposed models. The task of estimating parameters depends on the complexity of the proposed models and the method adopted to estimate them. Maximum likelihood estimation (MLE) is a popular method of parameter estimation but difficult to compute if the log-likelihood function of the corresponding model is too complicated. Random search methods for parameter optimization was first proposed by Brooks (1958). It is based on randomization of the parameter space and iteratively searching for optimal parameters by minimizing a criteria (e.x., sum of square errors). Exhaustive search for these parameters and all their combinations is clearly a limitation for models with more parameters, further, as these simulations are performed on a computer the numerical values of the parameter space are quantized which will lead to an approximate estimate of parameters. The pure random search method does not take the advantage of the local continuity properties of most criterion function (White 1971). 
In this paper, we propose a peak detection method to find certain parameters such as number of peaks and peak locations using wavelet transform modulus maxima which takes care of the local continuity. This method of estimating peak parameters will reduce the number of parameters that are to be searched randomly and provides exact estimates. The current procedure was experimented on spatial density model of February, 2010 TLE data set obtained from space-track website and the results are presented.

\section{Spatial density calculation}

The procedure adopted for calculating the spatial density is same as that reported by Anilkumar \& Sudheer Reddy (2009). Here, we outline the procedure to calculate the number of objects passing through the particular altitude bin [A km, B km] in a year, and use this to calculate the spatial density.

(i) Perigee filtering: The catalogued objects whose perigee is above $\mathrm{B} \mathrm{km}$ were filtered out.

(ii) Apogee filtering: The catalogued objects whose apogee is below A km were filtered out.

(iii) Other objects say ' $\mathrm{N}$ ' objects pass through the altitude bin [A km, B km]. Assume that in one orbit, the object crosses the bin 2 times on an average.

(iv) Number of objects in the bin in a year, NY $=2 * \mathrm{~N}^{*}$ norb, where norb is the number of times an object orbits the earth in a year, considering the repeated orbits of the objects in a year.

(v) Spatial Density $\mathrm{S}=\mathrm{NY} / \mathrm{V}$, where $\mathrm{V}$ is the volume of the annular region enclosed by the bin. $\mathrm{S}$ is in the unit of number of objects in a year per cubic $\mathrm{km}$.

These steps are executed on the two line element data set dated 4th Feb 2010. The spatial density calculated for this TLE in the inclination regions of $0-180,95-105,98-99^{\circ}$ from the altitude range of $200 \mathrm{~km}$ to $2000 \mathrm{~km}$ are shown in figures 3, 4 and 5, respectively.

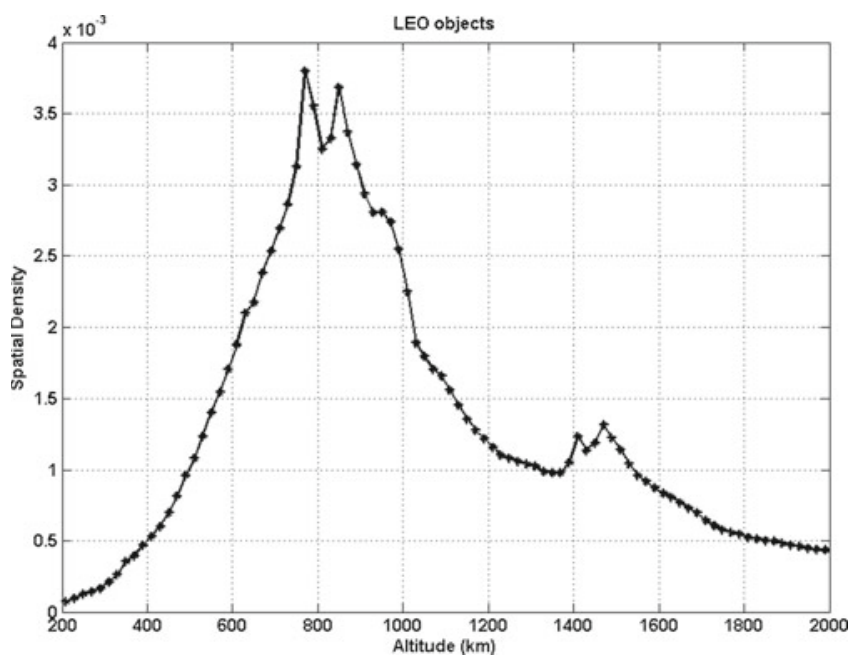

Figure 3. Spatial density at different altitudes in the inclination regions of $0-180^{\circ}$. 


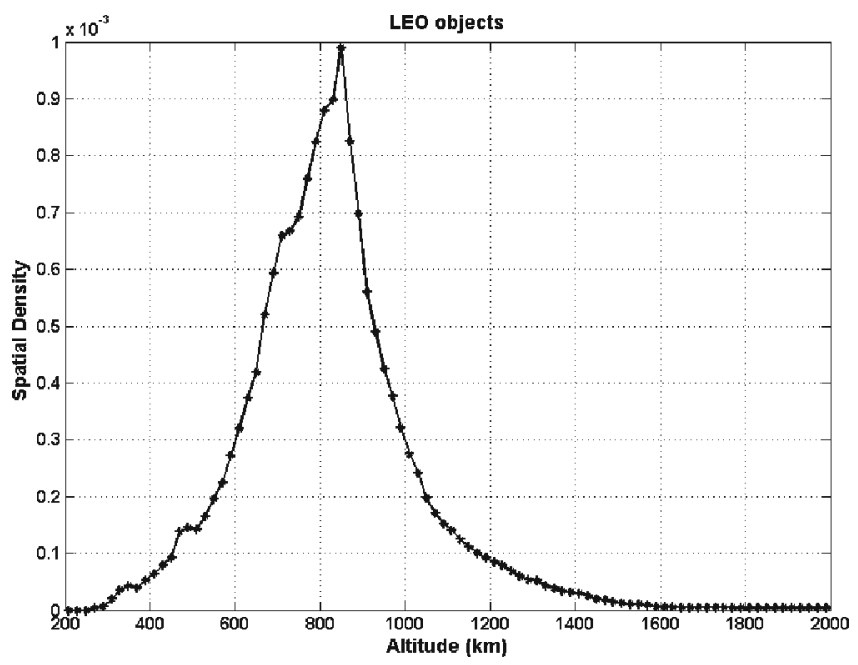

Figure 4. Spatial density at different altitudes in the inclination regions of $98-99^{\circ}$.

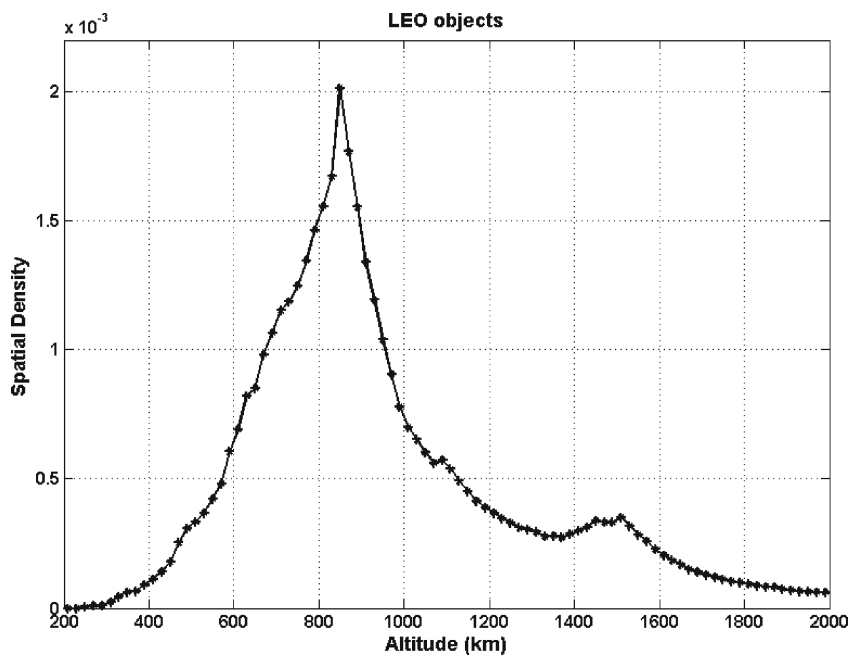

Figure 5. Spatial density at different altitudes in the inclination regions of $95-105^{\circ}$.

\section{Peak estimation using continuous wavelets}

A wavelet is a function $\psi(x)$ in $\mathrm{L}^{2}(\mathrm{R})$ such that $\psi(x)$ satisfies $\int_{-\infty}^{\infty} \psi(x) d x=0$. A CWT of a function $f(x)$ at point $\left(x_{0}, s\right)$ is defined as $W[f, \psi]_{\left(x_{0}, s\right)}=\left\langle f, \psi_{x_{0}, s}\right\rangle=$ $\frac{1}{\sqrt{s}} \int_{-\infty}^{\infty} f(x) \psi\left(\frac{x-x_{0}}{s}\right) d x$, where $\psi_{x_{0}, s}$ is $\psi(x)$ translated by $x_{0}$ and scaled by $s$, i.e., $\psi_{x_{0}, s}(x)=\frac{1}{\sqrt{s}} \psi\left(\frac{x-x_{0}}{s}\right)$. A wavelet transform modulus maxima is defined as a point $\left(x_{0}, s_{0}\right)$ 
such that $\left|W\left[f\left(x_{0}, s_{0}\right)\right]\right|$ is locally maximum at $x=x_{0}$, i.e., $\frac{\partial}{\partial x} W\left[f\left(x_{0}, s_{0}\right)\right]=0$. Any connected curve $\mathrm{s}(\mathrm{x})$ in the space-scale plane $(\mathrm{x}, \mathrm{s})$ along which all points are wavelet modulus maxima is called a maximal lines (Mallat 1998).

The CWT coefficients contain patterns of peaks and troughs which can be used to detect the position and strength of peaks in $f(x)$ of similar size to $\psi_{x_{0}, s}$. Varying $\mathrm{s}$ in $\psi_{x_{0}, s}$ will yield different-width wavelets, and so all peaks in $f(x)$, regardless of their width, can be detected. The accurate and local analysis of the functions/data requires redundant time and scale analyses. We choose the second derivative of Gaussian function $g(x)=c . e^{-x^{2}}$ called Mexican hat as analysing wavelet $\psi(x)=g^{(2)}(x)=\frac{2}{\pi^{1 / 4} \sqrt{3}}\left(x^{2}-1\right) \exp \left(\frac{-x^{2}}{2 \sigma^{2}}\right)$, normalized so that $\left\|g^{(2)}(x)\right\|=1$. These compact and continuously differentiable wavelets do not have an associated scaling function and thus have no filters, but have the advantage of having an explicit formula (Daubechies 1992). These properties provide us a method for peak detection using the continuous wavelet transform.

Mallat \& Hwang (1992) proved that if a signal $f(x)$ is singular at a point $x_{0}$, there exists a sequence of wavelet transform modulus maxima that converge to $x_{0}$ as the scale decreases $(s \rightarrow 0)$. This allows to detect all singularities from the position of the wavelet transform modulus maxima.

The modulus maxima of the wavelet coefficients produce connected curves at all scales of decomposition. Based on this fact, we propose an algorithm to detect singularities in the spatial density data, the outline of the algorithm is as follows:

(i) Initialize the choice of basis wavelet function $\psi$ and scale vector $s=\left[s_{1}, s_{2}, s_{3}, \ldots s_{m}\right]$ in ascending order.

(ii) Perform continuous wavelet transform on spatial density data $W=C W T(f(x))$. $W$ is a matrix of wavelet coefficients of size $\mathrm{M} \times \mathrm{N}$, where $\mathrm{M}$ is the number of scales and $\mathrm{N}$ is the length of data.

(iii) Find the positions of local maxima, say, $x_{m_{i}, s_{k}}$ for all scales $s_{k}, k=1,2,3 \ldots m$.

(iv) Remove all those $x_{m_{i}, s_{k}}$ at subsequent levels for $k=1,2,3 \ldots m$, for which $\left|x_{m_{i}, s_{k}}-x_{m_{i}, s_{k-1}}\right|>\tau$, where $\tau$ is a threshold corresponding to width of $\psi$ at scale $k$.

(v) Form a maximal line and count the number of levels in which the local maxima indices are in the neighbourhood of $x_{m_{i}, s_{k}} \pm \tau$ for each i across all scales.

(vi) Those $x_{m_{i}, s_{k}}$ are accepted as required peak locations for which the count is greater than a fixed number.

Maximal lines are used for removing false peaks if the length of lines are smaller than a given threshold. The width of a peak is proportional to the scale corresponding to the maximum amplitude on the ridge line. A peak candidate is dropped if its width is not in a given range.

\section{Modelling of the spatial density}

Since many of the earth observation satellites are in the sun synchronous orbits or near polar orbits the conjunction threat is more in this region, hence the inclination region of $95-105^{\circ}$ and $98-99^{\circ}$ are of special interest in addition to the conjunctions in overall inclination region of 0 $180^{\circ}$ at different altitude bands in LEO (Anilkumar \& Sudheer Reddy 2009). The calculated spatial densities are normalized so as to model with probability distributions.

Laplace distribution without area parameter was observed as the best suited distribution for modelling the number of objects in different altitude bands (Ananthasayanam et al 2006). The 
spatial density is modelled using modified Laplace distribution and mixtures of them (Anilkumar $\&$ Sudheer Reddy 2009). Here, we provide the modelling efforts carried out with TLE data of February, 2010. Functional form of Laplace distribution as a function of two parameters $m$ and $s$ is

$$
f(x)=\frac{1}{2 s} \exp \left(\frac{-|x-m|}{s}\right) .
$$

The parameter $m$ is called the location parameter and $s$ is called the scale parameter (Balakrishnan \& Nevzorov 2003). Modified form of Laplace distribution introducing one more parameter called area parameter ' $a$ ' is of the form

$$
f(x)=\frac{a}{2 s} \exp \left(\frac{-|x-m|}{s}\right) .
$$

The Binary mixture of modified Laplace distribution is

$$
f(x)=a\left(\frac{p}{2 s_{1}} \exp \left(\frac{-\left|x-m_{1}\right|}{s_{1}}\right)+\frac{(1-p)}{2 s_{2}} \exp \left(\frac{-\left|x-m_{2}\right|}{s_{2}}\right)\right),
$$

and, tertiary mixture,

$$
\begin{aligned}
f(x)= & a \cdot\left(p _ { 2 } \left(\frac{p_{1}}{2 s_{1}} \exp \left(\frac{-\left|x-m_{1}\right|}{s_{1}}\right)+\frac{\left(1-p_{1}\right)}{2 s_{2}}\right.\right. \\
& \left.\left.\times \exp \left(\frac{-\left|x-m_{2}\right|}{s_{2}}\right)\right)+\frac{\left(1-p_{2}\right)}{2 s_{3}} \exp \left(\frac{-\left|x-m_{3}\right|}{s_{3}}\right)\right) .
\end{aligned}
$$

The parameters for mixture of Laplace distributions are tuned by random search.

\section{Estimation of parameters based on wavelets and random search technique}

Parameter estimation falls in two categories deterministic and stochastic. The reliability of parameters obtained by either of the methods depends on the nature and complexity of the parametric model. Most of the deterministic parameter estimation methods rely on constructing an objective function and optimizing it using gradient methods. The scope of gradient dependent methods are limited for the objective functions that are not continuous and/or not differentiable. Hence, it is necessary to depend on probabilistic approaches such as random search, genetic algorithms, simulated annealing, etc., though they offer weak theoretical guarantee of convergence towards the global solution. In this section, we present simple random search method based on least square estimation, and our present methodology of peak parameter estimation using wavelets.

\subsection{Random search method}

Let $\left(x_{i}, y_{i}\right), i=1, \ldots, k$; where $k$ is the number of points in the given data. Let $z_{i}=$ $f\left(t_{i}, \theta_{j}, j=1 \ldots m\right)$ be the proposed model for the given data. The random search technique is adopted to search for those parameters which will minimize the sum of square errors, $S S E=$ $\sum_{i}\left(y_{i}-z_{i}\right)^{2}$, in a trial of large number of simulations (of the order of $10^{5}$ or more). Initially, we begin the search for the required $\theta_{j}\left(m_{1}, m_{2}, m_{3}, s_{1}, s_{2}, s_{3}, a, p_{1}, p_{2}\right)$ between the predefined lower limit $\theta_{L}$, and upper limit $\theta_{U}$ for each $j$. In these large number of simulations a sequence 
of parameter values $\theta_{j}$ for each $j$ are generated that converges to a value which gives minimum square error (Sorenson 1980).

The performance of random search method depends on the number of parameters, length of the initial search interval and number of simulations. The purpose of trying a large number of simulations is to achieve a possible minimum SSE. Probably this may not be the global minimum. Repeated experimenting with a simulated model in large number of trials we found that the variation between the parameters obtained by random search and the parameters corresponding to global minimum is not that alarming. Wavelets play an important role in fixing some of the parameters like number of peaks and peak locations. Conventionally this would have taken more time in fixing these parameters by random search method alone. Another advantage with this analysis is finding the exact position of true location parameters.

\subsection{Present methodology (wavelet-random search)}

The present procedure employs wavelet decomposition for locating peak position $\left(m_{i} ; i=1 . . m\right)$ and number of peaks $(m)$, on finding these peak parameters; we use random search method for estimating the area and scale parameters $\left(s_{i}, a\right)$. The present procedure is described in the following steps.

(i) Search for the peaks using wavelet transform which corresponds to location parameters $\left(m_{1}, m_{2}, m_{3}\right)$ using the criteria discussed above ( $\left.\sec 3\right)$.

(ii) Fix the location parameters of the model with these peak locations obtained in (i).

(iii) Initialize the starting search intervals for all parameters other than location parameters of the model $\left(s_{i}, p_{1}, p_{2}, a\right)$.

(iv) Find the optimal model parameters using random search method.

\section{Experimentation, results and discussions}

The proposed methodology is applied on the Two Line Element data sets of 4th February, 2010 obtained from space-track website (www.space-track.com), which contained 16,289 catalogued objects scanned on that day. As described in section 2, the spatial density for this data set was calculated as the number of objects per cubic kilometer residing in different altitude bands from $200 \mathrm{~km}$ to $2000 \mathrm{~km}$ with an interval of $20 \mathrm{~km}$, and three inclination regions $0^{\circ}-180^{\circ}, 95^{\circ}-105^{\circ}$, and $98^{\circ}-99^{\circ}$.

We chose second derivative of Gaussian called Mexican hat function as analysing wavelet. Continuous wavelet transform was operated on spatial density data of three inclination regions. Figures 6a, 7a and 8a show the wavelet spectrum corresponding to $0-180,98-99,95-105^{\circ}$, respectively. The propagation of singularities from higher scale to lower scales towards singularity can be clearly observed. Figures $6 \mathrm{~b}, 7 \mathrm{~b}$ and $8 \mathrm{~b}$ show the clustering of CWT coefficients around the singularities at all scales. The formation of maximal lines whose count is greater than 16 are shown in figures 9, 10 and 11 . Note the convergence of these maximal lines towards exact location of singularity across the scales. The values of singularities found were $850 \mathrm{~km}$ in $98-$ $99^{\circ}, 850 \mathrm{~km}, 1510 \mathrm{~km}$ in $95-105^{\circ}$, and $765 \mathrm{~km}, 850 \mathrm{~km}, 1470 \mathrm{~km}$ in $0-180^{\circ}$ inclinations. Once the singularities are established, the other parameters $\left(s_{1}, s_{2}, s_{3}, a, p_{1}, p_{2}\right)$ are tuned by random search method. The complexity of modulus maxima computation depends on the size of the data, for smaller one dimensional data such as spatial density it is not too expensive compared with any 2D data (e.g., digital images). The accurate location of the peak parameters using modulus maxima outweighs the method of locating them with pure random search. 

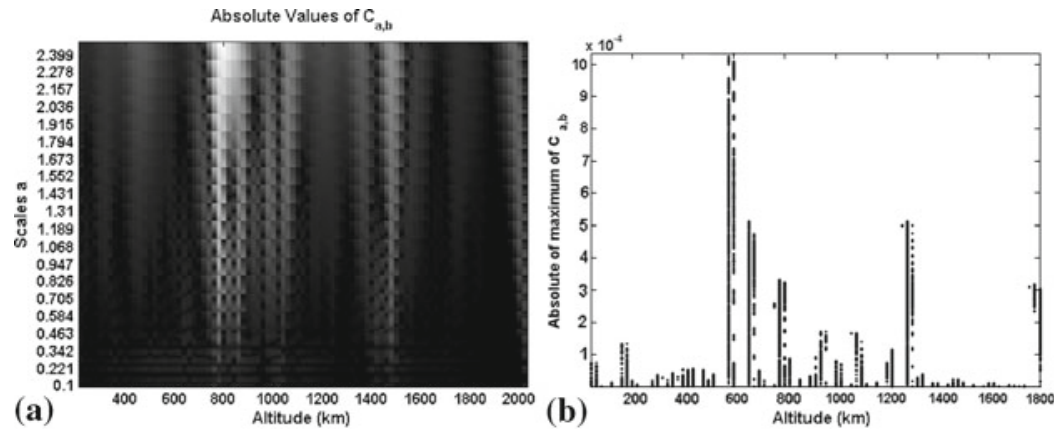

Figure 6. (a) Wavelet spectrum of spatial density in $0-180^{\circ}$ inclination. (b) Maximum modulus of coefficients of spatial density data in $0-180^{\circ}$ inclination.
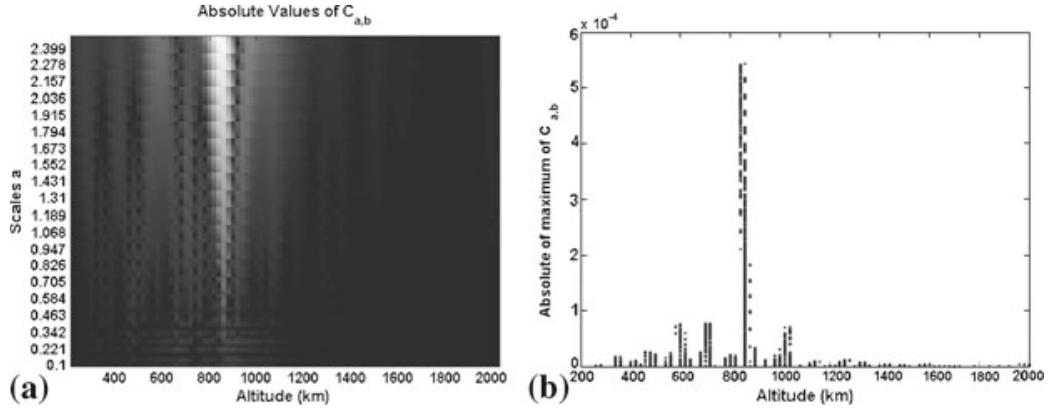

Figure 7. (a) Wavelet spectrum of spatial density in $98-99^{\circ}$ inclination. (b) Maximum modulus of coefficients of spatial density data in $98-99^{\circ}$ inclination.

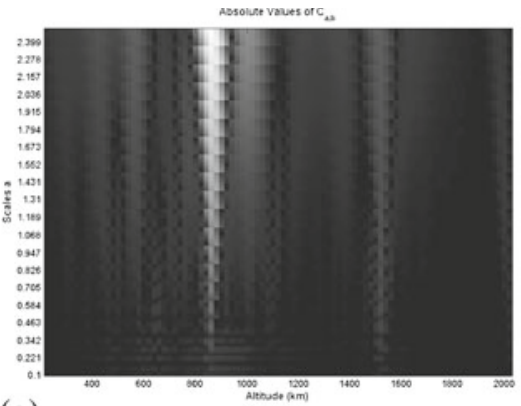

(a)

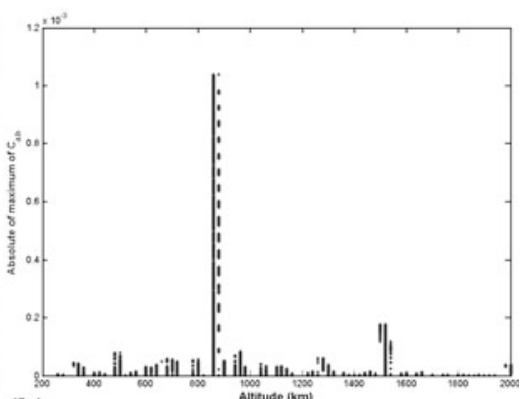

(b)

Figure 8. (a) Wavelet spectrum of spatial density in $95-105^{\circ}$ inclination. (b) Maximum modulus of coefficients of spatial density data in $95-105^{\circ}$ inclination. 


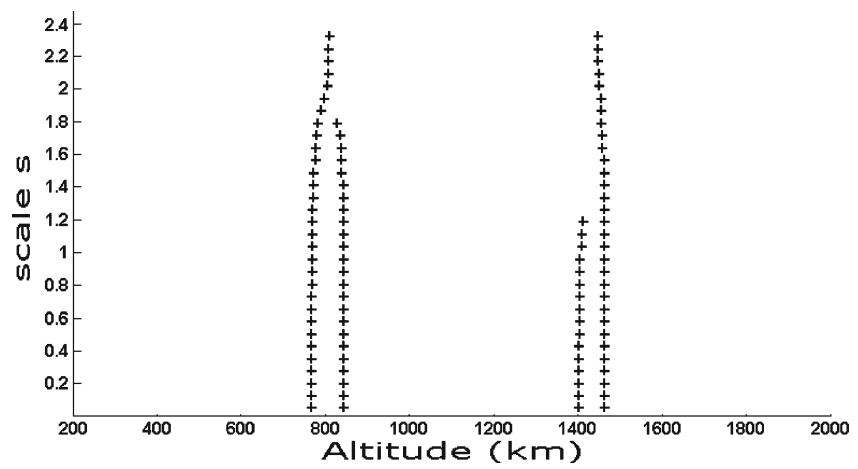

Figure 9. Maximal lines across different scales converging to singularity location for spatial density in $0-180^{\circ}$ inclination region.

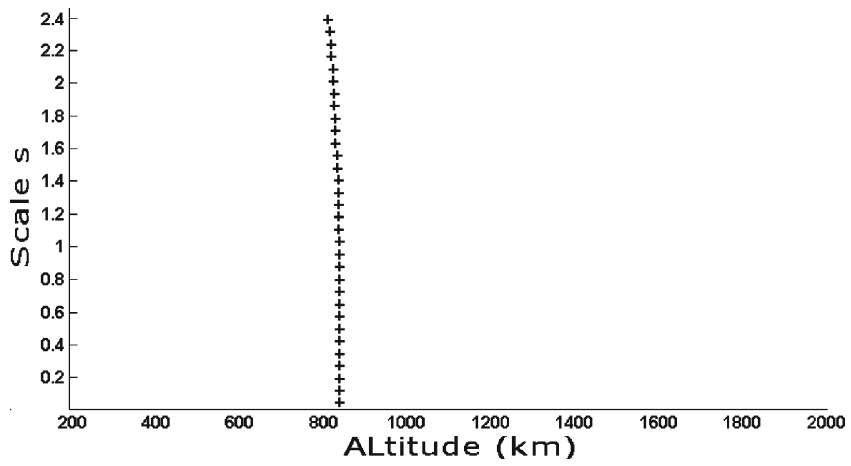

Figure 10. Maximal lines across different scales converging to singularity location for spatial density in 98-99 inclination region.

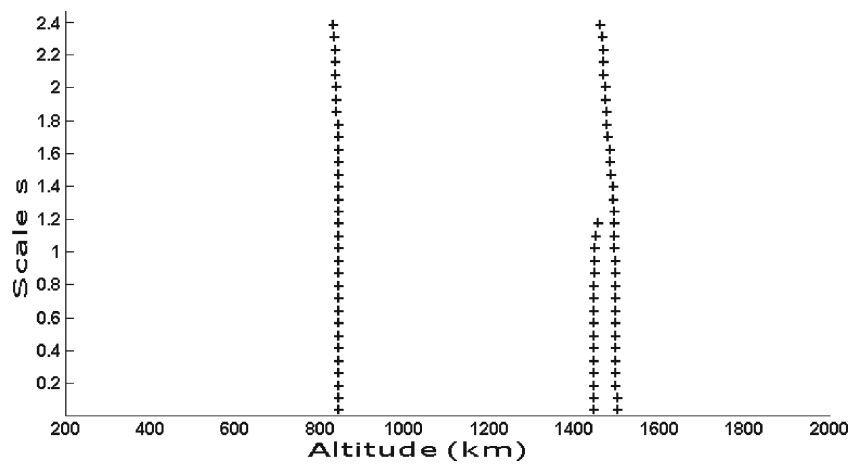

Figure 11. Maximal lines across different scales converging to singularity location for spatial density in $95-105^{\circ}$ inclination region. 
Table 1. Parameters in the inclination 98 to $99^{\circ}$.

\begin{tabular}{lccc}
\hline TLE sets & $\begin{array}{c}\text { Area } \\
\mathrm{a}\end{array}$ & $\begin{array}{c}\text { Location } \\
\text { M1 }\end{array}$ & $\begin{array}{c}\text { Scale } \\
\text { S1 }\end{array}$ \\
\hline Dec. 2006 & 13.163 & 800 & 111.3 \\
Aug. 2007 & 52.497 & 850 & 151.86 \\
Feb. 2010 & 61.05 & 850 & 169.0 \\
\hline
\end{tabular}

Table 2. Parameters in the inclination 0 to $180^{\circ}$.

\begin{tabular}{|c|c|c|c|c|c|c|c|c|c|}
\hline \multirow[b]{2}{*}{ TLE sets } & \multirow{2}{*}{$\frac{\text { Area(a) }}{a}$} & \multicolumn{3}{|c|}{ Location(M) } & \multicolumn{3}{|c|}{ Scale $(\mathrm{S})$} & \multicolumn{2}{|c|}{ Weights(P) } \\
\hline & & $\overline{\mathrm{M} 1}$ & M2 & M3 & S1 & S2 & S3 & $\mathrm{P} 1$ & $\mathrm{P} 2$ \\
\hline Dec. 2006 & 1.4121 & 900 & 1470 & - & 291.08 & 395.5 & - & 0.6 & - \\
\hline Aug. 2007 & 1.8706 & 850 & 1470 & - & 200.61 & 394.71 & - & 0.6 & - \\
\hline Feb. 2010 & 2.27 & 765 & 850 & 1470 & 199 & 57 & 317 & 0.89 & 0.65 \\
\hline
\end{tabular}

Table 3. Parameters in the inclination 95 to $105^{\circ}$.

\begin{tabular}{|c|c|c|c|c|c|c|c|c|c|}
\hline \multirow[b]{2}{*}{ TLE sets } & \multirow{2}{*}{$\frac{\text { Area(a) }}{a}$} & \multicolumn{3}{|c|}{ Location(M) } & \multicolumn{3}{|c|}{ Scale(S) } & \multicolumn{2}{|c|}{ Weights $(\mathrm{P})$} \\
\hline & & M1 & M2 & M3 & S1 & S2 & S3 & $\mathrm{P} 1$ & $\mathrm{P} 2$ \\
\hline Dec. 2006 & 5.3033 & 810 & 1090 & 1513.9 & 150.0 & 161.59 & 276.07 & 0.6999 & 0.5745 \\
\hline Aug. 2007 & 13.2 & 850 & 1470 & - & 167.1 & 254.80 & - & 0.80 & - \\
\hline Feb. 2010 & 14.96 & 850 & 1510 & - & 177 & 174 & - & 0.857 & - \\
\hline
\end{tabular}

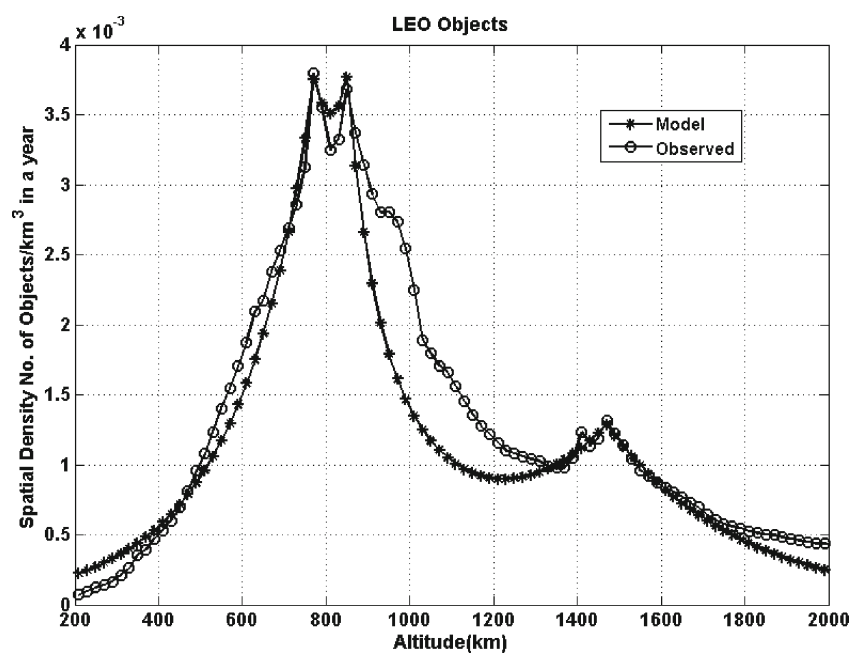

Figure 12. Fit of Laplace distribution for the spatial density data in the inclinations from $0-180^{\circ}$. 


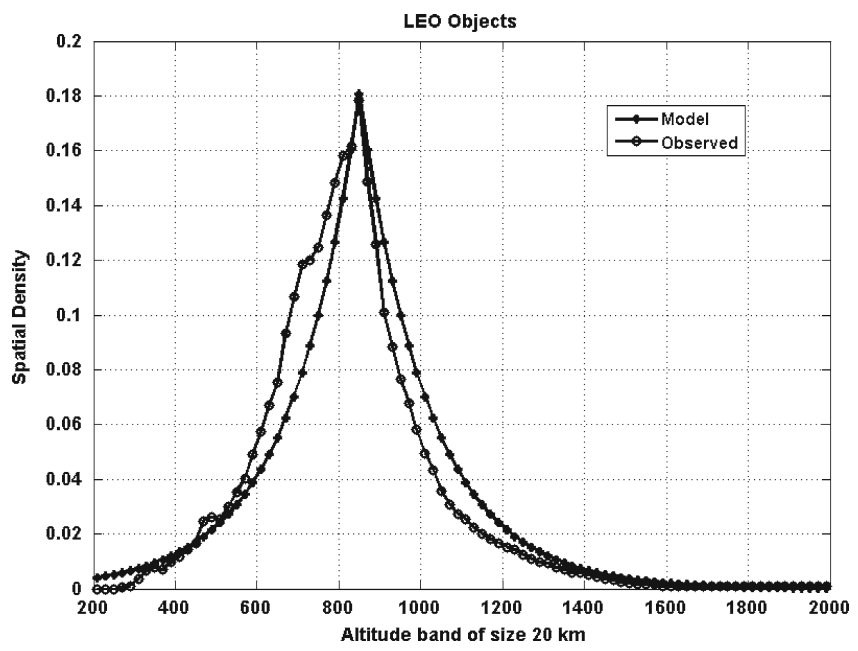

Figure 13. Fit of Laplace distribution for the spatial density data in the inclinations from $98-99^{\circ}$.

The lower and upper limit of initial search intervals $\left[\theta_{L}, \theta_{U}\right]$ for parameters $\mathrm{m}_{1}, \mathrm{~m}_{2}, \mathrm{~m}_{3}$ are chosen as $[200,2000], s_{1}, s_{2}, s_{3}$ are $[100,500], p_{1}, p_{2}$, are $[0.5,1]$. If $\mathrm{A}$ is the area under the data, then the limits for area parameter a are taken as $\left[0.8^{*} \mathrm{~A}, 1.2 * \mathrm{~A}\right]$, i.e., $\pm 20 \%$ of the area of $\mathrm{A}$.

The presence of noise in spatial density data is very mild and hence detection of false peaks is low. Tables 1, 2 and 3 display the model parameters thus obtained. The variation in peak locations found using purely random search method is around $\pm 4 \mathrm{~km}$. The values of parameters for 14th Dec, 2006 and 21st Aug, 2007 data sets are taken from Anilkumar \& Sudheer Reddy (2009) for comparison. The increasing trend of area parameter shows the growing number of space debris objects in turn implying the increasing number of conjunctions. Table 3 also indicates that the number of peaks changed from two in Aug 2007 to three in Feb 2010 in overall inclinations from

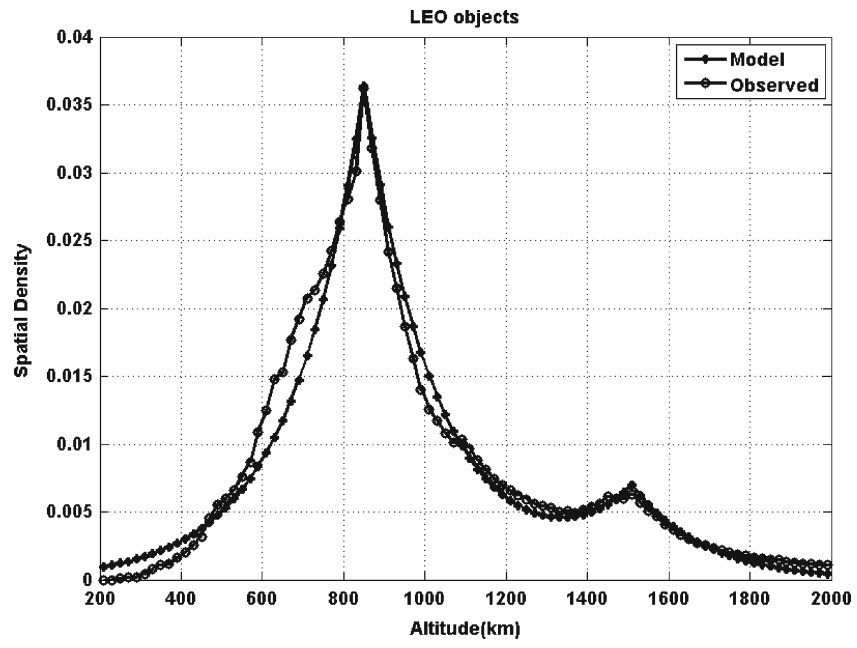

Figure 14. Fit of Laplace distribution for the spatial density data in the inclinations from $95-105^{\circ}$. 
$0^{\circ}-180^{\circ}$. Another peak formation at $765 \mathrm{~km}$ may be mainly attributed to the new explosions and collisions. The effect of third body perturbations, non-uniform Earth's gravitational field on the debris objects would also contribute for movement of the debris objects towards lower altitudes, but such a movement in a short span will be considerably small as reported in Sudheer Reddy \& Anilkumar (2008). Figures 12, 13 and 14 show the match between the model and the observed data.

\section{Conclusions}

A method based on continuous wavelets was proposed to find model parameters like peak number and peak position that will reduce the number of parameters to be estimated using random search method. This wavelet-based parameter estimation was found to be useful in finding exact location parameters of peaked distributions such as mixtures of Laplace distribution. The proposed methodology was applied on February 2010 TLE data. A new peak position at $765 \mathrm{~km}$ was observed in all inclinations from $0-180^{\circ}$ attributing to the new collisions and explosions that occurred during August 2007 to February 2010.

\section{References}

Ananthasayanam M R, Anilkumar A K and Subba Rao P V 2006 A new stochastic impressionistic low earth model of the space debris scenario. Acta Astro. 59(7): 547-559

Anilkumar A K and Sudheer Reddy D 2009 Statistical conjunction analysis for low-Earth-orbit catalogued objects. J. Spacecraft and Rockets, AIAA 160-167

Balakrishnan N and Nevzorov V B 2003 A primer on statistical distributions. Hoboken, New Jersey: John Wiley \& Sons. Inc

Brooks S H 1958 A discussion of random methods for seeking maxima. Operations Res. 6: 244-251

Daubechies I 1992 Ten lectures on wavelets. Society for Industrial and Applied Mathematics, Philadelphia Klinkrad H 2006 Space debris models and risk analysis. Chichester, UK: Springer-Praxis publishing Liou J C 2010 An updated assessment of the orbital debris environment in low Earth orbits. Orbital Debris Quarterly News 14(1): 7-8

Mallat S 1998 A wavelet tour of signal processing. San Diego: Academic Press

Mallat S and Hwang W L 1992 Singularity detection and processing with wavelets. IEEE Transactions on Information Theory, 38(2): 617-643

Orbital Debris Quarterly News (ODQN) website www.orbitaldebris.jsc.nasa.gov

Sorenson HW 1980 Parameter estimation - Principles and problems. New York: Marcel Dekker

Sudheer Reddy D and Anilkumar A K 2008 Analysis of conjunctions of low earth orbital debris considering drag and oblateness effects. Proc. of 53rd Cong. of ISTAM-08, 289-295

Vallado D A 2001 Fundamentals of astrodynamics and applications. Dordrecht: Kluwer Academic Press

White Jr R C 1971 A survey of random search methods for parameter optimization. Simulation 17(5): 197-205 